\title{
BMJ Open Anxiety and depression in Chinese patients with glaucoma and its correlations with vision-related quality of life and visual function indices: a cross-sectional study
}

Na Wu (1) , ${ }^{1,2,3}$ Xiangmei Kong, ${ }^{1,2,3}$ Xinghuai Sun ${ }^{1,2,3}$

To cite: Wu N, Kong X, Sun X. Anxiety and depression in Chinese patients with glaucoma and its correlations with vision-related quality of life and visual function indices: a crosssectional study. BMJ Open 2022;12:e046194. doi:10.1136/ bmjopen-2020-046194

- Prepublication history for this paper is available online. To view these files, please visit the journal online (http://dx.doi org/10.1136/bmjopen-2020046194).

Received 26 0ctober 2020 Accepted 26 January 2022

Check for updates

(C) Author(s) (or their employer(s)) 2022. Re-use permitted under CC BY-NC. No commercial re-use. See rights and permissions. Published by BMJ.

${ }^{1}$ Eye Institute and Department of Ophthalmology, Eye \& ENT Hospital, Fudan University, Shanghai, China

${ }^{2} \mathrm{NHC}$ Key Laboratory of Myopia (Fudan University); Key Laboratory of Myopia, Chinese Academy of Medical Sciences, Shanghai, China

${ }^{3}$ Shanghai Key Laboratory of Visual Impairment and Restoration, Shanghai, China

Correspondence to Dr Xiangmei Kong: kongxm95@163.com

\section{ABSTRACT}

Objective To investigate anxiety and depression in patients with glaucoma and its correlations with visionrelated quality of life (VR-QoL) and visual function indices. Design A hospital-based, cross-sectional study.

Setting Shanghai, China

Participants The Hospital Anxiety and Depression Scale and the 25-item National Eye Institute Visual Functioning Questionnaire (NEI VFQ-25) were administered to 446 Chinese patients with glaucoma to assess their psychological disorders, namely anxiety and depression, and VR-QoL, respectively.

Outcome measures Sociodemographic and clinical data were collected at the same time. Multivariate linear regression analysis was carried out to investigate the associations between VR-QoL, visual function indices and psychological disorders. Standardised partial regression analysis was used to identify the variable mostly indicative in evaluating psychological burdens.

Results The prevalence of anxiety and depression in Chinese patients with glaucoma was $12.11 \%$ and $25.78 \%$, respectively. Most of the subscales and the composite score of NEI VFQ-25 were negatively associated with each parameter of anxiety and depression after adjusting for sociodemographic and clinical variables. For visual function indices, only the best-corrected visual acuity of both eyes was correlated with anxiety after adjustment. Standardised partial regression analysis further showed VR-QoL, but not the objective visual function indices, was mostly relevant to psychological disturbances.

Conclusions Anxiety and depression were common in Chinese patients with glaucoma. Self-reported VR-QoL was beneficial in assessing psychological status of patients with glaucoma, while objective visual function indices provide little hint on it.

\section{INTRODUCTION}

Glaucoma is the leading cause of irreversible blindness globally. It was estimated that China would account for one quarter in number with glaucoma worldwide. ${ }^{1}$ Due to fear of blindness, increased financial burdens ${ }^{2}$ and long-term usage of anti-glaucoma medications, patients
Strengths and limitations of this study

- This is an initial Chinese study to investigate the effects of self-reported vision-related quality of life (VR-QoL) measured by 25-item National Eye Institute Visual Functioning Questionnaire and objective visual function indices on anxiety and depression in patients with glaucoma.

- Standardised partial regression analysis was used to find the most significant deciding factor for psychological disorders between VR-QoL and visual function indices.

- This study enrolled a relatively large number of participants with a broad age range and controlled for multiple confounding variables.

- The conclusions may not be generalised to the entire Chinese patients with glaucoma due to its nature of hospital-based study.

- The cross-sectional design of the study made it only assess association but not causality.

with glaucoma are more likely to suffer from psychological disturbances compared with the reference subjects. ${ }^{3}$ Among them, anxiety and depression are the two most common forms. ${ }^{3}$ Patients with glaucoma with depressive symptoms have a greater likelihood of developing poorer medication compliance. ${ }^{4}$

Glaucoma, as a chronic disease, has longstanding negative effects on patients' visionrelated quality of life (VR-QoL). ${ }^{5}$ Decreased QoL is related to increased economic burden on community and healthcare system. ${ }^{5}$ QoL has been increasingly considered as an important measure in glaucoma management. ${ }^{6}$ Anxiety and depression affect the VR-QoL of patients with glaucoma significantly. $^{78}$

Although a higher prevalence of anxiety and depression in patients with glaucoma, these disturbances have not raised enough clinical research interest. Only a few studies 
focused on anxiety, depression and their risk factors in patients with glaucoma. In this cross-sectional study, we investigated the associations between anxiety, depression and VR-QoL and objective visual function parameters including visual field (VF) and visual acuity (VA). In addition, we tried to find the most important influencing factor for psychological disorders in Chinese patients with glaucoma.

\section{METHODS}

Patients with glaucoma, including those with primary open-angle glaucoma (POAG), primary angle closure glaucoma (PACG) and secondary glaucoma (SG), were recruited consecutively at Eye \& ENT Hospital, Fudan University, in Shanghai, China. Diagnosis of POAG was based on typical glaucomatous disc cupping and compatible VF defects in at least one eye and with the presence of an open angle with a Shaffer grading of $>2$ on gonioscopy. PACG was diagnosed if there was at least two quadrants of closed angle where the trabecular meshwork was not visible on gonioscopy. Eyes with secondary causes of glaucoma were diagnosed as SG. Patients with other severe vision-impaired eye diseases, such as age-related macular degeneration, cataract (Lens Opacities Classification System III grade 2 or above), were not eligible to participate in this study. Patients who currently use medications that could have effects on psychiatric assessments were also excluded from the study, for example, systemic use of beta blockers. In addition, patients with VF defects caused by diseases other than glaucoma and patients who had incisional eye surgeries or laser treatments within the previous 3 months before the enrolment were not included in the study.

Questionnaires including a checklist of sociodemographic and clinical information, the Chinese versions of the Hospital Anxiety and Depression Scale (HADS) and the National Eye Institute Visual Function Questionnaire 25 (NEI VFQ-25) were distributed to the participants. A neutral interviewer would provide assistance to patients with poor sight or with illiteracy and record their choices. An independent hospital staff was responsible for assessing the data quality and the overall data management.

The HADS was composed of two subscales, representing HADS-anxiety (HADS-A) and HADS-depression (HADSD). It was developed by Zigmond and Snaith to identify and quantify anxiety and depression symptoms in populations with physical illnesses. ${ }^{9}$ Each subscale includes seven questions graded on a 4-point Likert scale from 0 to 3 . Thus, the minimum sum score for each subscale is 0 and the maximum is 21. Higher scores indicate higher degrees of anxiety and depression. As previously described, ${ }^{310}$ the scores of HADS-A and HADS-D above 10 were defined as anxiety and depression, respectively. The translated Chinese version from the original HADS questionnaire has been confirmed reliability and validity. ${ }^{211}$
The NEI VFQ-25 consists of 25 items related to visiontargeted QoL. These items are grouped into 12 subscales: general health, general vision, ocular pain, near activity, distance activity, social function, mental health, role difficulties, dependency, driving, colour vision and peripheral vision. Each subscale has a minimum value of 0 and a maximum value of 100 , indicating extreme disability and no disability, respectively. The composite score of the NEI VFQ-25 is the mean score of all the subscales except for general health domain.

All participants were underwent comprehensive ophthalmological examinations including the slit-lamp biomicroscopy. Best-corrected visual acuity (BCVA) was measured using the Snellen chart and was transformed to the logarithm of the minimum angle of resolution. Intraocular pressure (IOP) was measured by a Goldmann applanation tonometer. Cup-to-disc(C/D) ratio was evaluated according to the fundus photographs by an experienced ophthalmologist. A central $30^{\circ} \mathrm{VF}$ test was examined using the automated static perimetry (Humphrey Visual Field Analyzer 30-2, Humphrey Instruments, San Leandro, California, USA). VF tests taken within 3 months before the enrolment and with a reliability factor less than $15 \%$ were eligible for the study.

Descriptive statistics were calculated for all variables. Continuous variables were presented as the mean $\pm \mathrm{SD}$ and numbers and percentages were used to describe categorical variables. Pearson or Spearman test was used to assess the correlations between the variables and the psychological disorders. Student's t-test, non-parametric test or analysis of variance using Bonferroni post hoc test was carried out for comparisons of subgroups among variables. Multiple linear regression analysis was used to identify the predictive factors for psychological disturbances. Results were summarised as standardised partial regression coefficients. $\mathrm{P}$ value $<0.05$ was considered to be statistically significant.

\section{Patient and public involvement statement}

Patients or the public were not involved in the design, conduct, reporting and dissemination plans of our research.

\section{RESULTS}

Four hundred forty-six patients with glaucoma, including 247 POAG, 168 PACG and 31 SG patients, participated in the study. The sociodemographic and clinical information of the participants were shown in table 1 . The subjects consisted of 232 male and 214 female. The average age of the patients was $57.40 \pm 15.99($ mean \pm SD) years, ranging from 18 to 91 years. The mean better BCVA of both eyes was $0.20 \pm 0.29$ and $0.65 \pm 0.76$ in the worse eyes. The mean better MD of both eyes was $-7.54 \pm 6.07 \mathrm{~dB}$, whereas that of the worse eyes was $-13.93 \pm 8.26 \mathrm{~dB}$.

Figure 1 summarised the scores of the two questionnaires. The mean scores of the HADS-A and HADS-D in the subjects were $6.11 \pm 3.71$ (ranged from 0 to 18 , 
Table 1 Sociodemographic and clinical characteristics of the participants $(n=446)$

\begin{tabular}{|lc|}
\hline Variables & $\begin{array}{l}\text { Mean } \pm \text { SD } \\
\text { Range/number of } \\
\text { participants (\%) }\end{array}$ \\
\hline Male & $232(52.02 \%)$ \\
\hline Age, years & $57.40 \pm 15.99(18-91)$ \\
\hline $18-40$ & $79(17.7 \%)$ \\
\hline $41-60$ & $146(32.7 \%)$ \\
\hline$>60$ & $221(49.6 \%)$ \\
\hline Living with familyMarital status & $418(93.72 \%)$ \\
\hline Married & $401(89.91 \%)$ \\
\hline Divorced & $15(3.36 \%)$ \\
\hline Single & $30(6.73 \%)$ \\
\hline Education level & \\
\hline Primary school & $31(6.95 \%)$ \\
\hline Middle school & $228(51.12 \%)$ \\
\hline University and above & $187(41.93 \%)$ \\
\hline Type of glaucoma & \\
\hline POAG & $247(55.38 \%)$ \\
\hline PACG & $168(37.67 \%)$ \\
\hline SG & $31(6.95 \%)$ \\
\hline
\end{tabular}

Anti-glaucoma medications

\begin{tabular}{lc}
\hline 0 & $59(13.23 \%)$ \\
\hline 1 type & $160(35.87 \%)$ \\
\hline 2 types & $152(34.08 \%)$ \\
\hline 3 or more types & $75(16.82 \%)$ \\
\hline History of laser treatment & $66(14.8 \%)$ \\
\hline History of surgery treatment & $193(43.27 \%)$ \\
\hline Duration of glaucoma, years & $4.70 \pm 6.36(0.1-37)$ \\
\hline Better BCVA of both eyes & $0.20 \pm 0.29(-1.3$ to 2$)$ \\
\hline Worse BCVA of both eyes & $0.65 \pm 0.76(-0.17$ to 3.2$)$ \\
\hline Lower IOP of both eyes, $\mathrm{mm} \mathrm{Hg}$ & $16.00 \pm 5.13(6-53.7)$ \\
\hline Higher IOP of both eyes, $\mathrm{mm} \mathrm{Hg}$ & $20.40 \pm 9.23(7-65)$ \\
\hline Bigger C/D of both eyes & $0.73 \pm 0.20(0.1-1.0)$ \\
Smaller C/D of both eyes & $0.58 \pm 0.21(0.1-1.0)$ \\
\hline Better MD of both eyes, $\mathrm{dB}$ & $-7.54 \pm 6.07(-30.52$ to 0$)$ \\
Worse MD of both eyes, $\mathrm{dB}$ & $-13.93 \pm 8.26(-32.55$ to -0.34$)$ \\
\hline
\end{tabular}

BCVA, best-corrected visual acuity; C/D, cup-to-disc ratio; IOP, intraocular pressure; MD, mean deviation; PACG, primary angle closure glaucoma; POAG, primary open-angle glaucoma; SG, secondary glaucoma.

figure 1A) and 7.22 \pm 4.18 (ranged from 0 to 18 , figure 1A), respectively. As previously described, scores above 10 on the HADS-A and HADS-D were defined as anxiety and depression, respectively. ${ }^{10}$ In this study, the prevalence of anxiety and depression in patients with glaucoma was $12.11 \%$ and $25.78 \%$, respectively. The mean composite score of NEI VFQ-25 was $71.52 \pm 15.72$ (range: 14.79-97.17, figure 1B). Because of the high missing rate $(308 / 446,69.1 \%)$, driving had the lowest score among
A

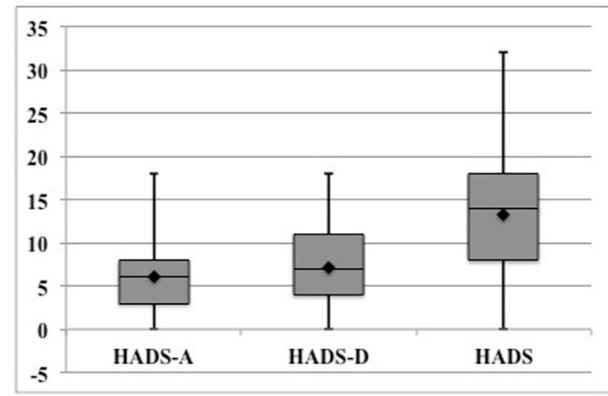

B

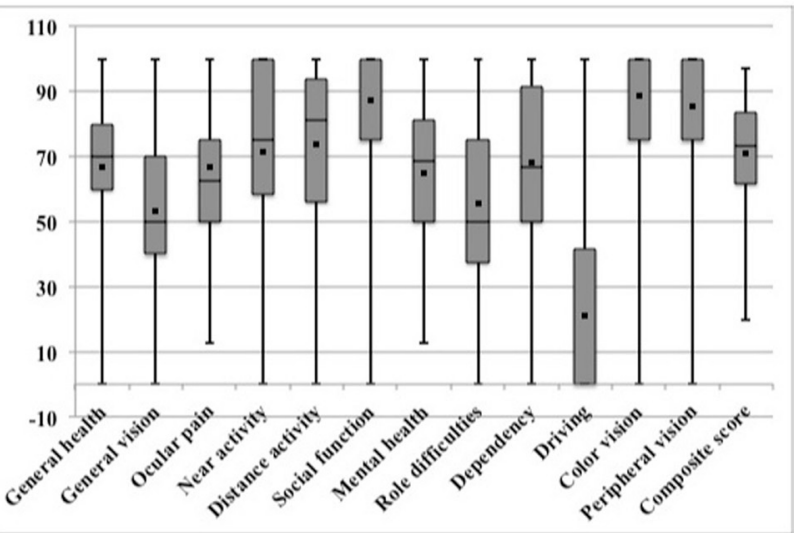

Figure 1 The average scores of the (A) HADS and the (B) NEI VFQ-25. Black squares and thin error bars represent means and maximum/minimum values, respectively. HADS-A, Hospital Anxiety and Depression Scale-Anxiety; HADS-D, Hospital Anxiety and Depression Scale-Depression; HADS, Hospital Anxiety and Depression Scale; NEI VFQ-25, National Eye Institute Visual Function Questionnaire 25.

the 12 subscales $(20.97 \pm 34.9$, figure $1 \mathrm{~B})$, while colour vision had the highest score $(88.62 \pm 21.74$, figure $1 \mathrm{~B})$. As previously reported, ${ }^{12}$ the subscale of driving was not included to calculate the composite score of NEI VQF-25. The univariate associations between sociodemographic, clinical factors and psychological disorders were shown in table 2. Age and educational level were both statistically significantly correlated with the scores of HADS-A and HADS-D (all $p<0.05)$. Living status $(p<0.001)$ and smaller $\mathrm{C} / \mathrm{D}$ of both eyes $(\mathrm{p}=0.007)$ were significantly correlated with anxiety disorder. Statistically significant correlations were observed between marital status $(\mathrm{p}=0.038)$, duration of glaucoma $(\mathrm{p}=0.004)$, type of glaucoma $(\mathrm{p}<0.001)$, numbers of anti-glaucoma medications $(\mathrm{p}<0.001)$, history of anti-glaucoma surgeries $(\mathrm{p}<0.001)$, higher IOP of both eyes $(p<0.001)$ and depression disorder. For visual function indices, better and worse BCVA of both eyes and better and worse MD of both eyes were all statistically significantly associated with the scores of HADS-A and HADS-D (all $\mathrm{p}<0.05)$. We further compared the differences in anxiety and depression based on the BCVA and MD stratifications. As shown in table 3, both the scores of HADS-A and HADS-D showed statistically significant differences among different levels of BCVA and MD in the better and the worse eyes (all overall $\mathrm{p}<0.05$ ).

The associations between the 12 domains and the composite score of NEI VFQ-25 and the psychological 
Open access

Table 2 Univariate comparisons between sociodemographic, clinical variables and the psychological disturbances

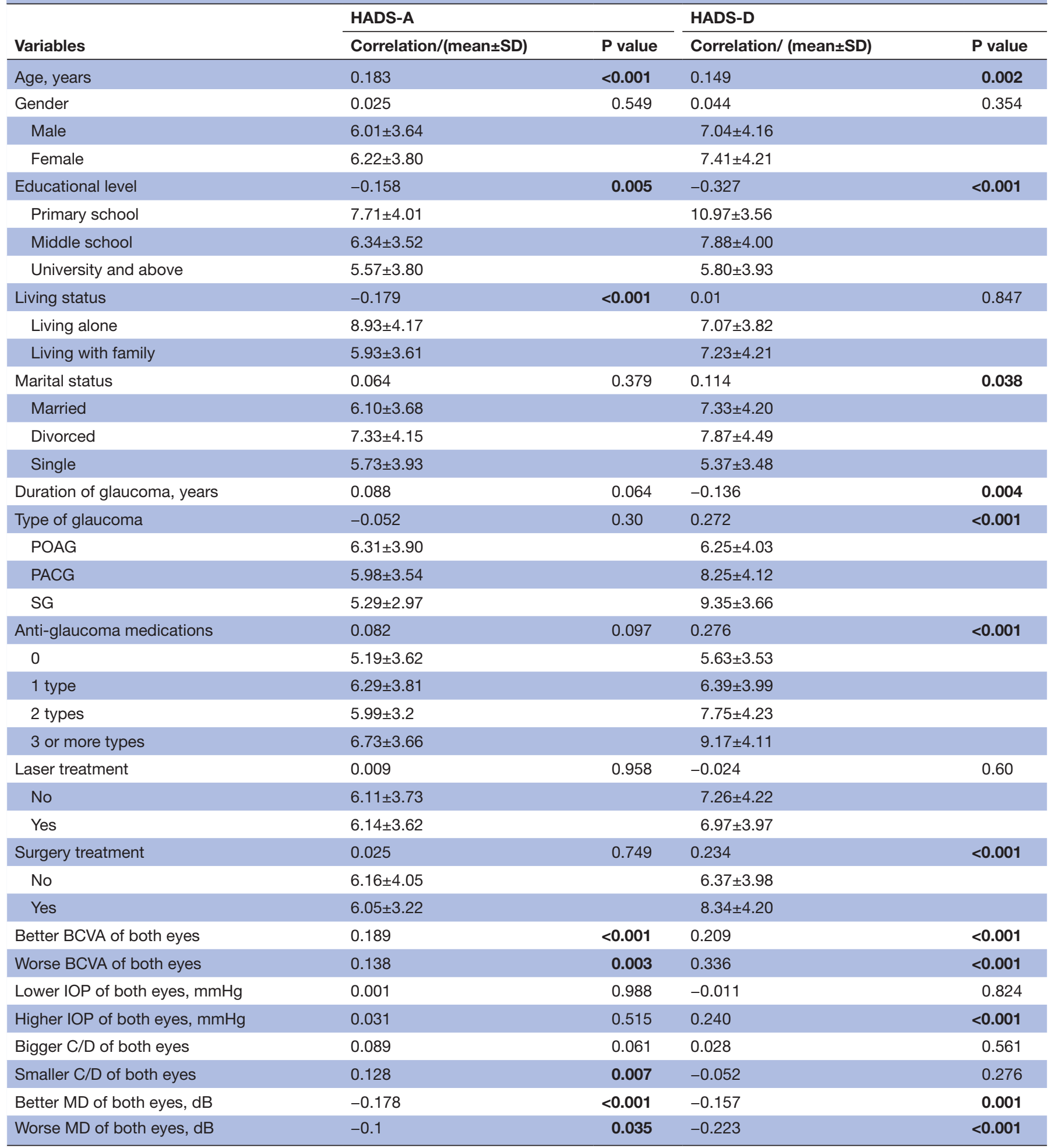

Bold $P$ values are $<0.05$ with statistical significances.

BCVA, best-corrected visual acuity; C/D, cup-to-disc ratio; HADS-A, Hospital Anxiety and Depression Scale-Anxiety; HADS-D, Hospital Anxiety and Depression Scale-Depression; IOP, intraocular pressure; MD, mean deviation; PACG, primary angle closure glaucoma; POAG, primary open-angle glaucoma; SG, secondary glaucoma.

disturbances by univariate analysis were shown in table 4 . All of the subscales and the total score of NEI VFQ-25 were significantly correlated with each parameter of anxiety and depression (all $\mathrm{p}<0.05$ ) except driving showed no statistical correlation with anxiety. 
Table 3 Comparisons of the HADS-A and HADS-D scores according to different levels of BCVA and MD

\begin{tabular}{|c|c|c|}
\hline Variables & $\begin{array}{l}\text { HADS-A } \\
\text { (mean } \pm S D)\end{array}$ & $\begin{array}{l}\text { HADS-D } \\
\text { (mean } \pm \text { SD) }\end{array}$ \\
\hline \multicolumn{3}{|l|}{ Better BCVA of both eyes } \\
\hline$>20 / 40(n=307)$ & $5.62 \pm 3.60$ & $6.62 \pm 4.14$ \\
\hline $20 / 200-20 / 40(n=134)$ & $7.12 \pm 3.76$ & $8.46 \pm 3.99$ \\
\hline$<20 / 200(n=5)$ & $9.40 \pm 1.95$ & $11.00 \pm 2.83$ \\
\hline Overall $p$ value & $<0.001$ & $<0.001$ \\
\hline \multicolumn{3}{|l|}{ Worse BCVA of both eyes } \\
\hline$>20 / 40(n=166)$ & $5.51 \pm 3.80$ & $5.62 \pm 3.88$ \\
\hline 20/200-20/40 (n=209) & $6.35 \pm 3.77$ & $7.63 \pm 3.94$ \\
\hline$<20 / 200(n=71)$ & $6.85 \pm 3.10$ & $9.75 \pm 4.06$ \\
\hline Overall $p$ value & 0.018 & $<0.001$ \\
\hline \multicolumn{3}{|l|}{ Better MD of both eyes } \\
\hline$>-10 \mathrm{~dB}(\mathrm{n}=336)$ & $5.77 \pm 3.63$ & $6.93 \pm 4.13$ \\
\hline-10 to $-20 \mathrm{~dB}(\mathrm{n}=86)$ & $7.30 \pm 3.89$ & $7.94 \pm 4.19$ \\
\hline$<-20 \mathrm{~dB}(\mathrm{n}=24)$ & $6.75 \pm 3.38$ & $8.67 \pm 4.44$ \\
\hline Overall $p$ value & 0.002 & 0.029 \\
\hline \multicolumn{3}{|l|}{ Worse MD of both eyes } \\
\hline$>-10 \mathrm{~dB}(\mathrm{n}=183)$ & $5.48 \pm 3.76$ & $6.19 \pm 3.86$ \\
\hline-10 to $-20 \mathrm{~dB}(\mathrm{n}=143)$ & $6.61 \pm 3.64$ & $7.57 \pm 4.21$ \\
\hline$<-20 \mathrm{~dB}(\mathrm{n}=120)$ & $6.50 \pm 3.61$ & $8.38 \pm 4.29$ \\
\hline Overall $p$ value & 0.01 & $<0.001$ \\
\hline
\end{tabular}

Bold $P$ values are $<0.05$ with statistical significances.

BCVA, best-corrected visual acuity; HADS-A, Hospital Anxiety and

Depression Scale-Anxiety; HADS-D, Hospital Anxiety and Depression Scale-Depression; MD, mean deviation.
Multivariate linear regression analysis was carried out to evaluate the relationships between VR-QoL, objective visual function indices and the psychological disturbances without or with adjustment for sociodemographic and clinical factors. The correlations between the 12 subscales, the composite score of NEI VFQ-25 and the psychological disorders were shown in figure 2. After adjustment for variables, the 11 dimensions (all $\mathrm{p}<0.01$, figure $2 \mathrm{~A}$ ) and the overall score of NEI VFQ-25 ( $\beta,-0.16 ; 95 \%$ CI -0.18 to $-0.14, \mathrm{p}<0.001$, figure $2 \mathrm{~A}$ ) were all negatively associated with HADS-A except driving showed positive correlation $(\beta, 0.01 ; 95 \%$ CI 0 to $0.02, p=0.048$, figure $2 A)$. Except dependency $(\mathrm{p}=0.187$, figure $2 \mathrm{~B})$ and peripheral vision $(\mathrm{p}=0.564$, figure $2 \mathrm{~B})$, the other dimensions (all $\mathrm{p}<0.01$, figure $2 \mathrm{~B}$ ) and the composite score of NEI VFQ-25 ( $\beta$, $-0.11 ; 95 \%$ CI -0.13 to $-0.08, \mathrm{p}<0.001$, figure $2 \mathrm{~B}$ ) showed negative correlations with HADS-D after adjustment. The effects of visual function components on anxiety and depression were shown in figure 3 . After adjustment, only better and worse BCVA of both eyes were significantly correlated with anxiety $(\beta,-1.24 ; 95 \%$ CI -2.32 to -0.16 , $\mathrm{p}=0.025$ and $\beta,-0.61 ; 95 \%$ CI -1.06 to $-0.16, p=0.008$, respectively, figure 3A). Neither BCVA in the better nor in the worse eyes showed statistical relationships with depression (both $p>0.05$, figure $3 \mathrm{~B}$ ). In addition, better and worse MD of both eyes had no statistically significant associations with each scale of HADS-A and HADS-D after adjustment (all $\mathrm{p}>0.05$, figure $3 \mathrm{~A}$ and $\mathrm{B}$ ).

Standardised partial regression analysis was further employed to identify which one played the most important role in deciding the psychological disturbances between the visual function indices and VR-QoL. As shown in

Table 4 Univariate comparisons between the 12 domains and the composite score of NEI VFQ-25 and the psychological disturbances

\begin{tabular}{|c|c|c|c|c|}
\hline \multirow[b]{2}{*}{ Variables } & \multicolumn{2}{|l|}{ HADS-A } & \multicolumn{2}{|l|}{ HADS-D } \\
\hline & Correlation coefficient & $P$ value & Correlation coefficient & $P$ value \\
\hline General health & -0.491 & $<0.001$ & -0.408 & $<0.001$ \\
\hline General vision & -0.406 & $<0.001$ & -0.423 & $<0.001$ \\
\hline Ocular pain & -0.582 & $<0.001$ & -0.299 & $<0.001$ \\
\hline Near activity & -0.371 & $<0.001$ & -0.493 & $<0.001$ \\
\hline Distance activity & -0.417 & $<0.001$ & -0.496 & $<0.001$ \\
\hline Social function & -0.421 & $<0.001$ & -0.269 & $<0.001$ \\
\hline Mental health & -0.552 & $<0.001$ & -0.254 & $<0.001$ \\
\hline Role difficulties & -0.479 & $<0.001$ & -0.269 & $<0.001$ \\
\hline Dependency & -0.197 & $<0.001$ & -0.145 & 0.002 \\
\hline Driving & -0.005 & 0.918 & -0.269 & $<0.001$ \\
\hline Colour vision & -0.397 & $<0.001$ & -0.307 & $<0.001$ \\
\hline Peripheral vision & -0.378 & $<0.001$ & -0.104 & 0.029 \\
\hline Composite score & -0.577 & $<0.001$ & -0.434 & $<0.001$ \\
\hline
\end{tabular}

HADS-A, Hospital Anxiety and Depression Scale-Anxiety; HADS-D, Hospital Anxiety and Depression Scale-Depression; NEI VFQ-25, National Eye Institute Visual Function Questionnaire 25. 
A

CRUDE

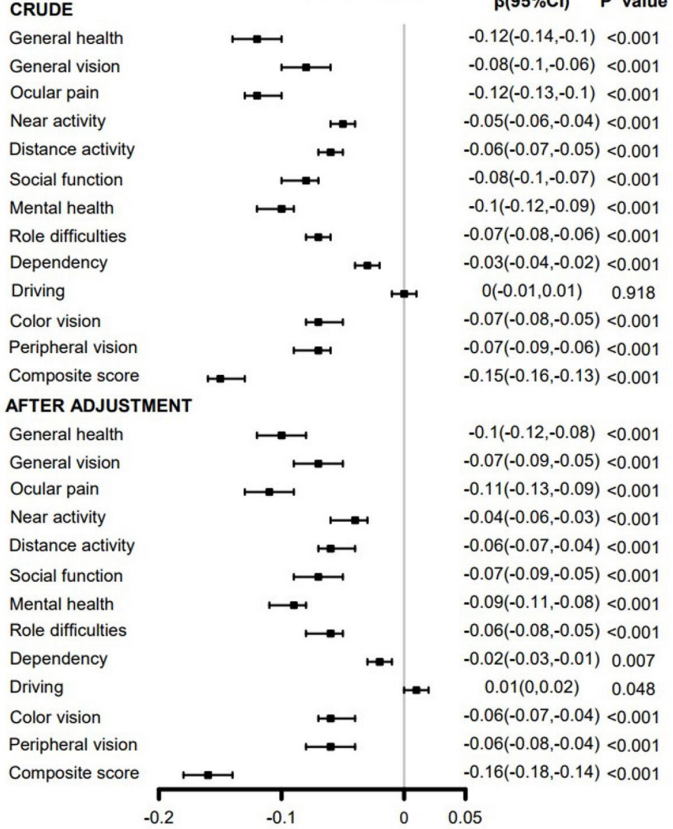

B

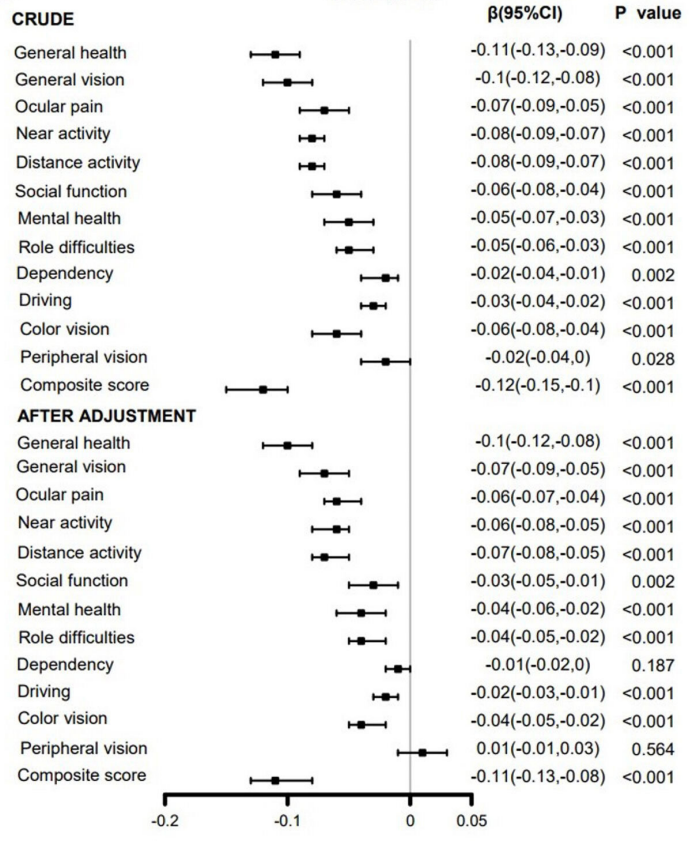

Figure 2 The effects of the subscales and the composite score of NEI VFQ-25 as independent variables on (A) HADS-A and (B) HADS-D by multivariate linear regression analysis without (CRUDE) or with adjustment (AFTER ADJUSTMENT) for sociodemographic and clinical variables. NEI VFQ-25, National Eye Institute Visual Function Questionnaire 25; HADS-A, Hospital Anxiety and Depression Scale-Anxiety; HADS-D, Hospital Anxiety and Depression Scale-Depression.

table 5, the composite score of NEI VFQ-25 was the only statistically significant variable in both anxiety and depression (both $\mathrm{p}<0.001$ ). The coefficient value of NEI VFQ-25 in HADS-A and HADS-D was -0.65 and -0.39 , respectively, which were the highest absolute values compared with the objective visual function indices.

\section{DISCUSSION}

The present study examined anxiety and depression in Chinese patients with glaucoma and investigated the factors influencing these two psychological disorders. The most important finding of the study was that patients' selfreported VR-QoL played the most important deciding role in psychiatric illnesses compared with objective visual function indices, such as MD and BCVA.

The prevalence of anxiety and depression in patients with glaucoma in our study was $12.11 \%$ and $25.78 \%$, respectively, which was significantly higher than that of the general normal Chinese population $(2.4 \%$ and $1.4 \%$ for anxiety and depression, respectively). It was consistent with previous studies, ${ }^{101314}$ indicating that glaucoma is a predictor of psychological disturbances. The prevalence of anxiety $(12.11 \%)$ in our study was significantly lower than that in Singapore $(64 \%)^{15}$ and was similar to that in Turkey $(13.5 \%)^{7}$ and Japan $(13 \%){ }^{10}$ The prevalence of depression $(25.78 \%)$ was lower than that in Turkey
A

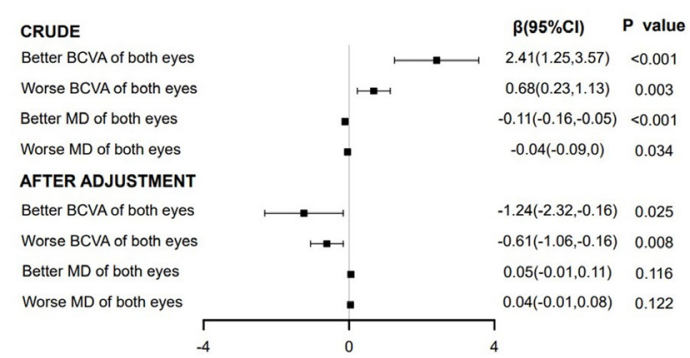

B

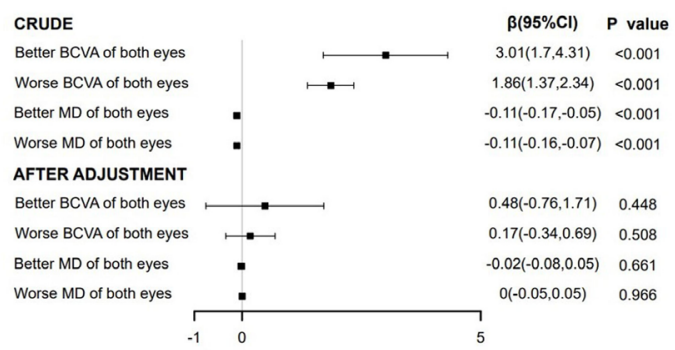

Figure 3 The effects of better and worse BCVA and better and worse MD of both eyes as independent variables on (A) HADS-A and (B) HADS-D by multivariate linear regression analysis without (CRUDE) or with adjustment (AFTER ADJUSTMENT) for sociodemographic, clinical variables and the composite score of NEI VFQ-25. BCVA, best-corrected visual acuity; MD, mean deviation; HADS-A, Hospital Anxiety and Depression Scale-Anxiety; HADS-D, Hospital Anxiety and Depression ScaleDepression; NEI VFQ-25, National Eye Institute Visual Function Questionnaire 25. 
Table 5 Results of standardised partial regression analysis to select the variable mostly relevant to HADS-A and HADS-D

\begin{tabular}{|c|c|c|c|c|c|c|}
\hline \multirow[b]{2}{*}{ Variables } & \multicolumn{3}{|l|}{ HADS-A } & \multicolumn{3}{|l|}{ HADS-D } \\
\hline & Regression coefficient & SE & $P$ value & Regression coefficient & SE & $P$ value \\
\hline Totalscore of NEI VFQ-25 & -0.65 & 0.01 & $<0.001$ & -0.39 & 0.01 & $<0.001$ \\
\hline Worse BCVA of both eyes & -0.10 & 0.25 & 0.07 & 0.03 & 0.29 & 0.59 \\
\hline Better MD of both eyes & 0.04 & 0.04 & 0.55 & -0.03 & 0.04 & 0.67 \\
\hline
\end{tabular}

HADS-A, Hospital Anxiety and Depression Scale-Anxiety; HADS-D, Hospital Anxiety and Depression Scale-Depression; NEI VFQ-25, the National Eye Institute Visual Function Questionnaire 25; BCVA, best-corrected visual acuity; MD, mean deviation.

$(57 \%)^{7}$ and Singapore $(30 \%),{ }^{15}$ but was higher than that in Australia $(19.09 \%),{ }^{16}$ Hungary $(12.1 \%){ }^{17}$ and America $(10.9 \%) .^{14}$

Univariate analysis showed that age had a positive but weak correlation with each parameter of anxiety and depression in patients with glaucoma. The relationship between age and anxiety is controversial. Zhang et al reported that the likelihood of having anxiety along with glaucoma did not change with age. ${ }^{18}$ However, other studies revealed that age was negatively correlated with anxiety. $^{2}{ }^{3}$ This inconsistency could be due to the differences in races, age ranges and numbers of patients with glaucoma included in these studies. In terms of depression, our findings were consistent with previous studies, ${ }^{3} 16$ which showed that older age was a risk factor for depression. Our results confirmed this finding in a larger span of ages (ranging from 18 to 91 years) in patients with glaucoma. Educational level was another demographic factor that reached statistical significance with both of anxiety and depression, which implied that increased educational level was along with decreased anxiety and depression symptoms. Living with family related to a decreased anxiety status. In addition, smaller C/D of both eyes was positively associated with anxiety, indicating that worse situation of the better eye signified a more serious anxiety status. A single marital status, long glaucoma course, POAG type, less numbers of glaucoma medications and no history of surgery treatment were related to a low degree of depression. Besides, higher IOP of both eyes was positively correlated with depression.

Multivariate linear regression analysis revealed that the composite score of NEI VFQ-25 and most of the 12 subscales were significantly negatively correlated with each parameter of anxiety and depression disorders after adjusting for sociodemographic and clinical variables. However, for visual function indices, only the better and the worse BCVA of both eyes were significantly related to anxiety after adjustment for other variables and the composite score of VR-QoL. Standardised partial regression analysis further showed that the composite score of NEI VFQ-25 accounted for the most important part in each scale of the HADS-A and HADS-D with statistical significance. Glaucoma is characterised by progressive optic neuropathy that could lead to visual function damage including VF defects and VA decrease. ${ }^{19}$ Ophthalmologists paid close attention to these objective visual function indices in clinical practice. However, our results indicated that instead of visual function components, patients' self-reported QoL played the most important role in psychological distresses.

Reports about the associations between self-reported measures, objective visual function indices and anxiety in eye diseases were very little. Zhou et al found that worse self-reported visual function assessed by Glaucoma Quality of Life-15 questionnaire (GQL-15) was consistently correlated with anxiety and depression in patients with glaucoma. ${ }^{2}$ In patients with retinitis pigmentosa, the degree of anxiety was significantly correlated with the general health and role difficulties of the NEI VFQ-25 dimensions, but not with any objective visual functions, such as better and worse BCVA of both eyes. ${ }^{20}$ With regard to depression, our results were consistent with several previous studies. Skalicky et al reported that the total score of GQL-15 was an independent predictor of depression. ${ }^{16}$ Jampel et al studied depression and mood indicators in newly diagnosed POAG patients. In compliance with our results, they found that poorer visual function, such as worse VA and more damaged VF, were not correlated with depression symptoms and altered mood. However, patients' perception of their vision in daily-life activities was associated with depression significantly. ${ }^{21}$ In a nationally representative glaucoma population, Wang et al observed that several self-reported measures of visual function including NEI VFQ-25 and how much time patients spent worrying about eyesight were significantly associated with depression. Objective measures of glaucoma severity, such as BCVA, MD and C/D, were not predictors of depression. ${ }^{14}$ Wilson et al also found that the severity of VA and VF were not predictors of depression. ${ }^{22}$

To our knowledge, this is an initial Chinese study to find that VR-QoL measured by NEI VFQ-25 was more informative in identifying patients with higher risk to suffer from psychological disorders. According to our results, patients with glaucoma with reduced VR-QoL are more vulnerable to develop anxiety and depression. On contrary, patients with good results of objective visual function measures, such as MD and BCVA, do not mean they have a healthy psychological status. Hence, patients 
with lower VR-QoL, but not with lower objective visual function performances, should be assessed for potential psychological disorders. This finding can help ophthalmologists and psychologists find patients with psychological disorders early. Psychological interventions from psychiatrists are needed for patients with glaucoma with severe psychological burdens if necessary. For ophthalmologists, except treating patients positively to save their objective visual functions, educating them an overall and detailed knowledge about glaucoma is essential and beneficial. For example, telling patients the slow progression nature of glaucoma and effectiveness in treatments on preventing glaucoma deterioration could alleviate their psychological abnormalities. Our previous study also confirmed that the levels on understanding about glaucoma is an independent factor negatively associated with anxiety and depression while positively associated with patients' VR-QoL. ${ }^{23}$

Our previous study showed psychological disorders, especially anxiety, impact VR-QoL more significantly compared with objective visual function indices in patients with glaucoma. ${ }^{8}$ Together with the present study, these two studies indicate that the subjective selfreported measures were more easily influenced by each other. Alleviating patients' psychological burdens is beneficial to improve their VR-QoL, and vice versa. These two studies provide a better understanding of the relationship between VR-QoL and psychological disturbances.

Caution should be exercised when interpreting the results due to several limitations. First, selection bias may be caused because the participants were recruited from a single tertiary institution. Our results may not be generalised to the entire Chinese patients with glaucoma due to its hospital-based design. Second, the cross-sectional nature of the study made it unable to establish causality. Longitudinal studies need to be performed to further confirm the conclusions. Third, self-reported measures may be affected by recall bias, thus may influence the interpretation of the results. Fourth, even though a multiple of confounding variables were analysed in the study, in no case were all potential factors included. In addition, even though the HADS questionnaire is commonly used for study purpose, it could not represent a formal psychological diagnosis of anxiety and depression. Despite the limitations, our study included a relatively large sample size with a broad age range, which may contribute to reducing bias.

In conclusion, our study showed that self-reported VR-QoL played the most important role in anxiety and depression disorders in patients with glaucoma. NEI VFQ-25 could be used as a useful tool to screen patients with glaucoma with high risk to suffer from psychological disturbances.

Acknowledgements We wish to thank all the participants for their time and invaluable devotion.

Contributors XK and XS conceived and designed the study. NW and XK collected the data, performed the statistical analysis, interpreted the data and drafted the manuscript. XK is responsible for the overall content as the guarantor. All authors contributed to the manuscript revision, read and approved the submitted version and the authorship list. XK is guarantor.

Funding This work was supported by the Surface Project of National Natural Science Foundation of China under Grant 81770922 and 82070957 ; the project of Shanghai Municipal Commission of Health and Family Planning under Grant 201740204; the clinical science and technology innovation project of Shanghai Shenkang Hospital Development Center under Grant SHDC12017X18; the western medicine guidance project of Shanghai Committee of Science and Technology under Grant 19411961600; Experimental Animal Research Project of Shanghai Science and Technology Commission under Grant 201409006600 and Double Excellence Project of Eye \& ENT Hospital of Fudan University under Grant SYB202003.

Disclaimer The funders had no role in study design, data collection and analysis, decision to publish or preparation of the manuscript.

Competing interests None declared.

Patient and public involvement Patients and/or the public were not involved in the design, or conduct, or reporting, or dissemination plans of this research.

Patient consent for publication Not applicable.

Ethics approval This study involves human participants and was approved by the Ethics Committee of Eye \& ENT Hospital, Fudan University. Participants gave informed consent to participate in the study before taking part.

Provenance and peer review Not commissioned; externally peer reviewed.

Data availability statement No data are available. No additional data are available.

Open access This is an open access article distributed in accordance with the Creative Commons Attribution Non Commercial (CC BY-NC 4.0) license, which permits others to distribute, remix, adapt, build upon this work non-commercially, and license their derivative works on different terms, provided the original work is properly cited, appropriate credit is given, any changes made indicated, and the use is non-commercial. See: http://creativecommons.org/licenses/by-nc/4.0/.

ORCID iD

Na Wu http://orcid.org/0000-0001-6485-9656

\section{REFERENCES}

1 Quigley HA, Broman AT. The number of people with glaucoma worldwide in 2010 and 2020. Br J Ophthalmol 2006;90:262-7.

2 Zhou C, Qian S, Wu P, et al. Anxiety and depression in Chinese patients with glaucoma: sociodemographic, clinical, and selfreported correlates. J Psychosom Res 2013;75:75-82.

3 Mabuchi F, Yoshimura K, Kashiwagi K, et al. Risk factors for anxiety and depression in patients with glaucoma. Br J Ophthalmol 2012;96:821-5.

4 Jayawant SS, Bhosle MJ, Anderson RT, et al. Depressive symptomatology, medication persistence, and associated healthcare costs in older adults with glaucoma. J Glaucoma 2007;16:513-20.

5 Mills T, Law SK, Walt J, et al. Quality of life in glaucoma and three other chronic diseases: a systematic literature review. Drugs Aging 2009;26:933-50.

6 Onakoya AO, Mbadugha CA, Aribaba OT, et al. Quality of life of primary open angle glaucoma patients in Lagos, Nigeria: clinical and sociodemographic correlates. J Glaucoma 2012;21:287-95.

7 Tastan S, Iyigun E, Bayer A, et al. Anxiety, depression, and quality of life in Turkish patients with glaucoma. Psychol Rep 2010;106:343-57.

$8 \mathrm{Wu} \mathrm{N}, \mathrm{Kong} \mathrm{X}, \mathrm{Gao} \mathrm{J}$, et al. Vision-related quality of life in glaucoma patients and its correlations with psychological disturbances and visual function indices. J Glaucoma 2019;28:207-15.

9 Zigmond AS, Snaith RP. The hospital anxiety and depression scale. Acta Psychiatr Scand 1983;67:361-70.

10 Mabuchi F, Yoshimura K, Kashiwagi K, et al. High prevalence of anxiety and depression in patients with primary open-angle glaucoma. J Glaucoma 2008;17:552-7.

11 Leung CM, Ho S, Kan CS, et al. Evaluation of the Chinese version of the hospital anxiety and depression scale. A cross-cultural perspective. Int J Psychosom 1993;40:29-34.

12 Suzukamo Y, Oshika T, Yuzawa M, et al. Psychometric properties of the 25-item National eye Institute visual function questionnaire (Nei VFQ-25), Japanese version. Health Qual Life Outcomes 2005;3:65. 
13 Chan EW, Chiang PPC, Liao J, et al. Glaucoma and associated visual acuity and field loss significantly affect glaucoma-specific psychosocial functioning. Ophthalmology 2015;122:494-501.

14 Wang SY, Singh K, Lin SC. Prevalence and predictors of depression among participants with glaucoma in a nationally representative population sample. Am J Ophthalmol 2012;154:436-44.

15 Lim NCS, Fan CHJ, Yong MKH, et al. Assessment of depression, anxiety, and quality of life in Singaporean patients with glaucoma. $J$ Glaucoma 2016;25:605-12.

16 Skalicky S, Goldberg I. Depression and quality of life in patients with glaucoma: a cross-sectional analysis using the geriatric depression Scale-15, assessment of function related to vision, and the glaucoma quality of Life-15. J Glaucoma 2008;17:546-51.

17 Holló G, Kóthy P, Géczy A, et al. Personality traits, depression, and objectively measured adherence to once-daily prostaglandin analog medication in glaucoma. J Glaucoma 2009;18:288-92.

18 Zhang $\mathrm{X}$, Olson DJ, Le $\mathrm{P}$, et al. The association between glaucoma, anxiety, and depression in a large population. Am J Ophthalmol 2017;183:37-41.
19 Yuki K, Awano-Tanabe S, Ono T, et al. Risk factors for motor vehicle collisions in patients with primary open-angle glaucoma: a multicenter prospective cohort study. PLoS One 2016;11:e0166943.

20 Sainohira M, Yamashita T, Terasaki H, et al. Quantitative analyses of factors related to anxiety and depression in patients with retinitis pigmentosa. PLoS One 2018;13:e0195983.

21 Jampel HD, Frick KD, Janz NK, et al. Depression and mood indicators in newly diagnosed glaucoma patients. Am J Ophthalmol 2007;144:238-44.

22 Wilson MR, Coleman AL, Yu F, et al. Depression in patients with glaucoma as measured by self-report surveys. Ophthalmology 2002;109:1018-22.

23 Kong XM, Zhu WQ, Hong JX, et al. Is glaucoma comprehension associated with psychological disturbance and vision-related quality of life for patients with glaucoma? A cross-sectional study. BMJ Open 2014;4:e004632. 